\title{
The Distributivity on Bi-Approximation Semantics
}

\author{
Tomoyuki Suzuki
}

\begin{abstract}
In this paper, we give a possible characterization of the distributivity on bi-approximation semantics. To this end, we introduce new notions of special elements on polarities and show that the distributivity is first-order definable on bi-approximation semantics. In addition, we investigate the dual representation of those structures and compare them with bi-approximation semantics for intuitionistic logic. We also discuss that two different methods to validate the distributivity - by the splitters and by the adjointness — can be explicated with the help of the axiom of choice as well.
\end{abstract}

\section{Introduction}

Bi-approximation semantics is a universal relational-type semantics for substructural and lattice-based logics, not necessarily including distributive substructural logics such as orthonormal logic or lattice-based modal logics (see Suzuki [11]). Unlike other relational semantics for nondistributive lattice-based logics (see, e.g., Goldblatt [7], Hartonas [8], Hartonas and Dunn [9], Gehrke [5]; see also Restall [10]), the novelty of bi-approximation semantics is to reason not only about formulas but also sequents, that is, logical consequences, based on polarities. As bi-approximation semantics was introduced to explicate Ghilardi and Meloni's [6] canonicity methodology via relational-type structures, we can enjoy the canonicity results of latticebased logics in Suzuki [12] (cf. residuated frames in Galatos and Jipsen [3]). In other words, we may say that bi-approximation semantics is a canonicity-friendly relational semantics for lattice-based logics. In addition, a Sahlqvist-type first-order definability for substructural logic was already shown in Suzuki [14]. Therefore, this completes the so-called Sahlqvist theorem for substructural and lattice-based logics.

Received April 23, 2013; accepted May 12, 2014

First published online April 20, 2016

2010 Mathematics Subject Classification: Primary 03G10, 03G25; Secondary 03G27

Keywords: lattice-based logics, relational semantics, canonicity

(C) 2016 by University of Notre Dame 10.1215/00294527-3542442 


\title{
The Distributivity on Bi-Approximation Semantics
}

\author{
Tomoyuki Suzuki
}

\begin{abstract}
In this paper, we give a possible characterization of the distributivity on bi-approximation semantics. To this end, we introduce new notions of special elements on polarities and show that the distributivity is first-order definable on bi-approximation semantics. In addition, we investigate the dual representation of those structures and compare them with bi-approximation semantics for intuitionistic logic. We also discuss that two different methods to validate the distributivity - by the splitters and by the adjointness — can be explicated with the help of the axiom of choice as well.
\end{abstract}

\section{Introduction}

Bi-approximation semantics is a universal relational-type semantics for substructural and lattice-based logics, not necessarily including distributive substructural logics such as orthonormal logic or lattice-based modal logics (see Suzuki [11]). Unlike other relational semantics for nondistributive lattice-based logics (see, e.g., Goldblatt [7], Hartonas [8], Hartonas and Dunn [9], Gehrke [5]; see also Restall [10]), the novelty of bi-approximation semantics is to reason not only about formulas but also sequents, that is, logical consequences, based on polarities. As bi-approximation semantics was introduced to explicate Ghilardi and Meloni's [6] canonicity methodology via relational-type structures, we can enjoy the canonicity results of latticebased logics in Suzuki [12] (cf. residuated frames in Galatos and Jipsen [3]). In other words, we may say that bi-approximation semantics is a canonicity-friendly relational semantics for lattice-based logics. In addition, a Sahlqvist-type first-order definability for substructural logic was already shown in Suzuki [14]. Therefore, this completes the so-called Sahlqvist theorem for substructural and lattice-based logics.

Received April 23, 2013; accepted May 12, 2014

First published online April 20, 2016

2010 Mathematics Subject Classification: Primary 03G10, 03G25; Secondary 03G27

Keywords: lattice-based logics, relational semantics, canonicity

(C) 2016 by University of Notre Dame 10.1215/00294527-3542442 


\title{
The Distributivity on Bi-Approximation Semantics
}

\author{
Tomoyuki Suzuki
}

\begin{abstract}
In this paper, we give a possible characterization of the distributivity on bi-approximation semantics. To this end, we introduce new notions of special elements on polarities and show that the distributivity is first-order definable on bi-approximation semantics. In addition, we investigate the dual representation of those structures and compare them with bi-approximation semantics for intuitionistic logic. We also discuss that two different methods to validate the distributivity - by the splitters and by the adjointness — can be explicated with the help of the axiom of choice as well.
\end{abstract}

\section{Introduction}

Bi-approximation semantics is a universal relational-type semantics for substructural and lattice-based logics, not necessarily including distributive substructural logics such as orthonormal logic or lattice-based modal logics (see Suzuki [11]). Unlike other relational semantics for nondistributive lattice-based logics (see, e.g., Goldblatt [7], Hartonas [8], Hartonas and Dunn [9], Gehrke [5]; see also Restall [10]), the novelty of bi-approximation semantics is to reason not only about formulas but also sequents, that is, logical consequences, based on polarities. As bi-approximation semantics was introduced to explicate Ghilardi and Meloni's [6] canonicity methodology via relational-type structures, we can enjoy the canonicity results of latticebased logics in Suzuki [12] (cf. residuated frames in Galatos and Jipsen [3]). In other words, we may say that bi-approximation semantics is a canonicity-friendly relational semantics for lattice-based logics. In addition, a Sahlqvist-type first-order definability for substructural logic was already shown in Suzuki [14]. Therefore, this completes the so-called Sahlqvist theorem for substructural and lattice-based logics.

Received April 23, 2013; accepted May 12, 2014

First published online April 20, 2016

2010 Mathematics Subject Classification: Primary 03G10, 03G25; Secondary 03G27

Keywords: lattice-based logics, relational semantics, canonicity

(C) 2016 by University of Notre Dame 10.1215/00294527-3542442 


\title{
The Distributivity on Bi-Approximation Semantics
}

\author{
Tomoyuki Suzuki
}

\begin{abstract}
In this paper, we give a possible characterization of the distributivity on bi-approximation semantics. To this end, we introduce new notions of special elements on polarities and show that the distributivity is first-order definable on bi-approximation semantics. In addition, we investigate the dual representation of those structures and compare them with bi-approximation semantics for intuitionistic logic. We also discuss that two different methods to validate the distributivity - by the splitters and by the adjointness — can be explicated with the help of the axiom of choice as well.
\end{abstract}

\section{Introduction}

Bi-approximation semantics is a universal relational-type semantics for substructural and lattice-based logics, not necessarily including distributive substructural logics such as orthonormal logic or lattice-based modal logics (see Suzuki [11]). Unlike other relational semantics for nondistributive lattice-based logics (see, e.g., Goldblatt [7], Hartonas [8], Hartonas and Dunn [9], Gehrke [5]; see also Restall [10]), the novelty of bi-approximation semantics is to reason not only about formulas but also sequents, that is, logical consequences, based on polarities. As bi-approximation semantics was introduced to explicate Ghilardi and Meloni's [6] canonicity methodology via relational-type structures, we can enjoy the canonicity results of latticebased logics in Suzuki [12] (cf. residuated frames in Galatos and Jipsen [3]). In other words, we may say that bi-approximation semantics is a canonicity-friendly relational semantics for lattice-based logics. In addition, a Sahlqvist-type first-order definability for substructural logic was already shown in Suzuki [14]. Therefore, this completes the so-called Sahlqvist theorem for substructural and lattice-based logics.

Received April 23, 2013; accepted May 12, 2014

First published online April 20, 2016

2010 Mathematics Subject Classification: Primary 03G10, 03G25; Secondary 03G27

Keywords: lattice-based logics, relational semantics, canonicity

(C) 2016 by University of Notre Dame 10.1215/00294527-3542442 


\title{
The Distributivity on Bi-Approximation Semantics
}

\author{
Tomoyuki Suzuki
}

\begin{abstract}
In this paper, we give a possible characterization of the distributivity on bi-approximation semantics. To this end, we introduce new notions of special elements on polarities and show that the distributivity is first-order definable on bi-approximation semantics. In addition, we investigate the dual representation of those structures and compare them with bi-approximation semantics for intuitionistic logic. We also discuss that two different methods to validate the distributivity - by the splitters and by the adjointness — can be explicated with the help of the axiom of choice as well.
\end{abstract}

\section{Introduction}

Bi-approximation semantics is a universal relational-type semantics for substructural and lattice-based logics, not necessarily including distributive substructural logics such as orthonormal logic or lattice-based modal logics (see Suzuki [11]). Unlike other relational semantics for nondistributive lattice-based logics (see, e.g., Goldblatt [7], Hartonas [8], Hartonas and Dunn [9], Gehrke [5]; see also Restall [10]), the novelty of bi-approximation semantics is to reason not only about formulas but also sequents, that is, logical consequences, based on polarities. As bi-approximation semantics was introduced to explicate Ghilardi and Meloni's [6] canonicity methodology via relational-type structures, we can enjoy the canonicity results of latticebased logics in Suzuki [12] (cf. residuated frames in Galatos and Jipsen [3]). In other words, we may say that bi-approximation semantics is a canonicity-friendly relational semantics for lattice-based logics. In addition, a Sahlqvist-type first-order definability for substructural logic was already shown in Suzuki [14]. Therefore, this completes the so-called Sahlqvist theorem for substructural and lattice-based logics.

Received April 23, 2013; accepted May 12, 2014

First published online April 20, 2016

2010 Mathematics Subject Classification: Primary 03G10, 03G25; Secondary 03G27

Keywords: lattice-based logics, relational semantics, canonicity

(C) 2016 by University of Notre Dame 10.1215/00294527-3542442 


\title{
The Distributivity on Bi-Approximation Semantics
}

\author{
Tomoyuki Suzuki
}

\begin{abstract}
In this paper, we give a possible characterization of the distributivity on bi-approximation semantics. To this end, we introduce new notions of special elements on polarities and show that the distributivity is first-order definable on bi-approximation semantics. In addition, we investigate the dual representation of those structures and compare them with bi-approximation semantics for intuitionistic logic. We also discuss that two different methods to validate the distributivity - by the splitters and by the adjointness — can be explicated with the help of the axiom of choice as well.
\end{abstract}

\section{Introduction}

Bi-approximation semantics is a universal relational-type semantics for substructural and lattice-based logics, not necessarily including distributive substructural logics such as orthonormal logic or lattice-based modal logics (see Suzuki [11]). Unlike other relational semantics for nondistributive lattice-based logics (see, e.g., Goldblatt [7], Hartonas [8], Hartonas and Dunn [9], Gehrke [5]; see also Restall [10]), the novelty of bi-approximation semantics is to reason not only about formulas but also sequents, that is, logical consequences, based on polarities. As bi-approximation semantics was introduced to explicate Ghilardi and Meloni's [6] canonicity methodology via relational-type structures, we can enjoy the canonicity results of latticebased logics in Suzuki [12] (cf. residuated frames in Galatos and Jipsen [3]). In other words, we may say that bi-approximation semantics is a canonicity-friendly relational semantics for lattice-based logics. In addition, a Sahlqvist-type first-order definability for substructural logic was already shown in Suzuki [14]. Therefore, this completes the so-called Sahlqvist theorem for substructural and lattice-based logics.

Received April 23, 2013; accepted May 12, 2014

First published online April 20, 2016

2010 Mathematics Subject Classification: Primary 03G10, 03G25; Secondary 03G27

Keywords: lattice-based logics, relational semantics, canonicity

(C) 2016 by University of Notre Dame 10.1215/00294527-3542442 


\title{
The Distributivity on Bi-Approximation Semantics
}

\author{
Tomoyuki Suzuki
}

\begin{abstract}
In this paper, we give a possible characterization of the distributivity on bi-approximation semantics. To this end, we introduce new notions of special elements on polarities and show that the distributivity is first-order definable on bi-approximation semantics. In addition, we investigate the dual representation of those structures and compare them with bi-approximation semantics for intuitionistic logic. We also discuss that two different methods to validate the distributivity - by the splitters and by the adjointness — can be explicated with the help of the axiom of choice as well.
\end{abstract}

\section{Introduction}

Bi-approximation semantics is a universal relational-type semantics for substructural and lattice-based logics, not necessarily including distributive substructural logics such as orthonormal logic or lattice-based modal logics (see Suzuki [11]). Unlike other relational semantics for nondistributive lattice-based logics (see, e.g., Goldblatt [7], Hartonas [8], Hartonas and Dunn [9], Gehrke [5]; see also Restall [10]), the novelty of bi-approximation semantics is to reason not only about formulas but also sequents, that is, logical consequences, based on polarities. As bi-approximation semantics was introduced to explicate Ghilardi and Meloni's [6] canonicity methodology via relational-type structures, we can enjoy the canonicity results of latticebased logics in Suzuki [12] (cf. residuated frames in Galatos and Jipsen [3]). In other words, we may say that bi-approximation semantics is a canonicity-friendly relational semantics for lattice-based logics. In addition, a Sahlqvist-type first-order definability for substructural logic was already shown in Suzuki [14]. Therefore, this completes the so-called Sahlqvist theorem for substructural and lattice-based logics.

Received April 23, 2013; accepted May 12, 2014

First published online April 20, 2016

2010 Mathematics Subject Classification: Primary 03G10, 03G25; Secondary 03G27

Keywords: lattice-based logics, relational semantics, canonicity

(C) 2016 by University of Notre Dame 10.1215/00294527-3542442 


\title{
The Distributivity on Bi-Approximation Semantics
}

\author{
Tomoyuki Suzuki
}

\begin{abstract}
In this paper, we give a possible characterization of the distributivity on bi-approximation semantics. To this end, we introduce new notions of special elements on polarities and show that the distributivity is first-order definable on bi-approximation semantics. In addition, we investigate the dual representation of those structures and compare them with bi-approximation semantics for intuitionistic logic. We also discuss that two different methods to validate the distributivity - by the splitters and by the adjointness — can be explicated with the help of the axiom of choice as well.
\end{abstract}

\section{Introduction}

Bi-approximation semantics is a universal relational-type semantics for substructural and lattice-based logics, not necessarily including distributive substructural logics such as orthonormal logic or lattice-based modal logics (see Suzuki [11]). Unlike other relational semantics for nondistributive lattice-based logics (see, e.g., Goldblatt [7], Hartonas [8], Hartonas and Dunn [9], Gehrke [5]; see also Restall [10]), the novelty of bi-approximation semantics is to reason not only about formulas but also sequents, that is, logical consequences, based on polarities. As bi-approximation semantics was introduced to explicate Ghilardi and Meloni's [6] canonicity methodology via relational-type structures, we can enjoy the canonicity results of latticebased logics in Suzuki [12] (cf. residuated frames in Galatos and Jipsen [3]). In other words, we may say that bi-approximation semantics is a canonicity-friendly relational semantics for lattice-based logics. In addition, a Sahlqvist-type first-order definability for substructural logic was already shown in Suzuki [14]. Therefore, this completes the so-called Sahlqvist theorem for substructural and lattice-based logics.

Received April 23, 2013; accepted May 12, 2014

First published online April 20, 2016

2010 Mathematics Subject Classification: Primary 03G10, 03G25; Secondary 03G27

Keywords: lattice-based logics, relational semantics, canonicity

(C) 2016 by University of Notre Dame 10.1215/00294527-3542442 


\title{
The Distributivity on Bi-Approximation Semantics
}

\author{
Tomoyuki Suzuki
}

\begin{abstract}
In this paper, we give a possible characterization of the distributivity on bi-approximation semantics. To this end, we introduce new notions of special elements on polarities and show that the distributivity is first-order definable on bi-approximation semantics. In addition, we investigate the dual representation of those structures and compare them with bi-approximation semantics for intuitionistic logic. We also discuss that two different methods to validate the distributivity - by the splitters and by the adjointness — can be explicated with the help of the axiom of choice as well.
\end{abstract}

\section{Introduction}

Bi-approximation semantics is a universal relational-type semantics for substructural and lattice-based logics, not necessarily including distributive substructural logics such as orthonormal logic or lattice-based modal logics (see Suzuki [11]). Unlike other relational semantics for nondistributive lattice-based logics (see, e.g., Goldblatt [7], Hartonas [8], Hartonas and Dunn [9], Gehrke [5]; see also Restall [10]), the novelty of bi-approximation semantics is to reason not only about formulas but also sequents, that is, logical consequences, based on polarities. As bi-approximation semantics was introduced to explicate Ghilardi and Meloni's [6] canonicity methodology via relational-type structures, we can enjoy the canonicity results of latticebased logics in Suzuki [12] (cf. residuated frames in Galatos and Jipsen [3]). In other words, we may say that bi-approximation semantics is a canonicity-friendly relational semantics for lattice-based logics. In addition, a Sahlqvist-type first-order definability for substructural logic was already shown in Suzuki [14]. Therefore, this completes the so-called Sahlqvist theorem for substructural and lattice-based logics.

Received April 23, 2013; accepted May 12, 2014

First published online April 20, 2016

2010 Mathematics Subject Classification: Primary 03G10, 03G25; Secondary 03G27

Keywords: lattice-based logics, relational semantics, canonicity

(C) 2016 by University of Notre Dame 10.1215/00294527-3542442 


\title{
The Distributivity on Bi-Approximation Semantics
}

\author{
Tomoyuki Suzuki
}

\begin{abstract}
In this paper, we give a possible characterization of the distributivity on bi-approximation semantics. To this end, we introduce new notions of special elements on polarities and show that the distributivity is first-order definable on bi-approximation semantics. In addition, we investigate the dual representation of those structures and compare them with bi-approximation semantics for intuitionistic logic. We also discuss that two different methods to validate the distributivity - by the splitters and by the adjointness — can be explicated with the help of the axiom of choice as well.
\end{abstract}

\section{Introduction}

Bi-approximation semantics is a universal relational-type semantics for substructural and lattice-based logics, not necessarily including distributive substructural logics such as orthonormal logic or lattice-based modal logics (see Suzuki [11]). Unlike other relational semantics for nondistributive lattice-based logics (see, e.g., Goldblatt [7], Hartonas [8], Hartonas and Dunn [9], Gehrke [5]; see also Restall [10]), the novelty of bi-approximation semantics is to reason not only about formulas but also sequents, that is, logical consequences, based on polarities. As bi-approximation semantics was introduced to explicate Ghilardi and Meloni's [6] canonicity methodology via relational-type structures, we can enjoy the canonicity results of latticebased logics in Suzuki [12] (cf. residuated frames in Galatos and Jipsen [3]). In other words, we may say that bi-approximation semantics is a canonicity-friendly relational semantics for lattice-based logics. In addition, a Sahlqvist-type first-order definability for substructural logic was already shown in Suzuki [14]. Therefore, this completes the so-called Sahlqvist theorem for substructural and lattice-based logics.

Received April 23, 2013; accepted May 12, 2014

First published online April 20, 2016

2010 Mathematics Subject Classification: Primary 03G10, 03G25; Secondary 03G27

Keywords: lattice-based logics, relational semantics, canonicity

(C) 2016 by University of Notre Dame 10.1215/00294527-3542442 


\title{
The Distributivity on Bi-Approximation Semantics
}

\author{
Tomoyuki Suzuki
}

\begin{abstract}
In this paper, we give a possible characterization of the distributivity on bi-approximation semantics. To this end, we introduce new notions of special elements on polarities and show that the distributivity is first-order definable on bi-approximation semantics. In addition, we investigate the dual representation of those structures and compare them with bi-approximation semantics for intuitionistic logic. We also discuss that two different methods to validate the distributivity - by the splitters and by the adjointness — can be explicated with the help of the axiom of choice as well.
\end{abstract}

\section{Introduction}

Bi-approximation semantics is a universal relational-type semantics for substructural and lattice-based logics, not necessarily including distributive substructural logics such as orthonormal logic or lattice-based modal logics (see Suzuki [11]). Unlike other relational semantics for nondistributive lattice-based logics (see, e.g., Goldblatt [7], Hartonas [8], Hartonas and Dunn [9], Gehrke [5]; see also Restall [10]), the novelty of bi-approximation semantics is to reason not only about formulas but also sequents, that is, logical consequences, based on polarities. As bi-approximation semantics was introduced to explicate Ghilardi and Meloni's [6] canonicity methodology via relational-type structures, we can enjoy the canonicity results of latticebased logics in Suzuki [12] (cf. residuated frames in Galatos and Jipsen [3]). In other words, we may say that bi-approximation semantics is a canonicity-friendly relational semantics for lattice-based logics. In addition, a Sahlqvist-type first-order definability for substructural logic was already shown in Suzuki [14]. Therefore, this completes the so-called Sahlqvist theorem for substructural and lattice-based logics.

Received April 23, 2013; accepted May 12, 2014

First published online April 20, 2016

2010 Mathematics Subject Classification: Primary 03G10, 03G25; Secondary 03G27

Keywords: lattice-based logics, relational semantics, canonicity

(C) 2016 by University of Notre Dame 10.1215/00294527-3542442 


\title{
The Distributivity on Bi-Approximation Semantics
}

\author{
Tomoyuki Suzuki
}

\begin{abstract}
In this paper, we give a possible characterization of the distributivity on bi-approximation semantics. To this end, we introduce new notions of special elements on polarities and show that the distributivity is first-order definable on bi-approximation semantics. In addition, we investigate the dual representation of those structures and compare them with bi-approximation semantics for intuitionistic logic. We also discuss that two different methods to validate the distributivity - by the splitters and by the adjointness — can be explicated with the help of the axiom of choice as well.
\end{abstract}

\section{Introduction}

Bi-approximation semantics is a universal relational-type semantics for substructural and lattice-based logics, not necessarily including distributive substructural logics such as orthonormal logic or lattice-based modal logics (see Suzuki [11]). Unlike other relational semantics for nondistributive lattice-based logics (see, e.g., Goldblatt [7], Hartonas [8], Hartonas and Dunn [9], Gehrke [5]; see also Restall [10]), the novelty of bi-approximation semantics is to reason not only about formulas but also sequents, that is, logical consequences, based on polarities. As bi-approximation semantics was introduced to explicate Ghilardi and Meloni's [6] canonicity methodology via relational-type structures, we can enjoy the canonicity results of latticebased logics in Suzuki [12] (cf. residuated frames in Galatos and Jipsen [3]). In other words, we may say that bi-approximation semantics is a canonicity-friendly relational semantics for lattice-based logics. In addition, a Sahlqvist-type first-order definability for substructural logic was already shown in Suzuki [14]. Therefore, this completes the so-called Sahlqvist theorem for substructural and lattice-based logics.

Received April 23, 2013; accepted May 12, 2014

First published online April 20, 2016

2010 Mathematics Subject Classification: Primary 03G10, 03G25; Secondary 03G27

Keywords: lattice-based logics, relational semantics, canonicity

(C) 2016 by University of Notre Dame 10.1215/00294527-3542442 


\title{
The Distributivity on Bi-Approximation Semantics
}

\author{
Tomoyuki Suzuki
}

\begin{abstract}
In this paper, we give a possible characterization of the distributivity on bi-approximation semantics. To this end, we introduce new notions of special elements on polarities and show that the distributivity is first-order definable on bi-approximation semantics. In addition, we investigate the dual representation of those structures and compare them with bi-approximation semantics for intuitionistic logic. We also discuss that two different methods to validate the distributivity - by the splitters and by the adjointness — can be explicated with the help of the axiom of choice as well.
\end{abstract}

\section{Introduction}

Bi-approximation semantics is a universal relational-type semantics for substructural and lattice-based logics, not necessarily including distributive substructural logics such as orthonormal logic or lattice-based modal logics (see Suzuki [11]). Unlike other relational semantics for nondistributive lattice-based logics (see, e.g., Goldblatt [7], Hartonas [8], Hartonas and Dunn [9], Gehrke [5]; see also Restall [10]), the novelty of bi-approximation semantics is to reason not only about formulas but also sequents, that is, logical consequences, based on polarities. As bi-approximation semantics was introduced to explicate Ghilardi and Meloni's [6] canonicity methodology via relational-type structures, we can enjoy the canonicity results of latticebased logics in Suzuki [12] (cf. residuated frames in Galatos and Jipsen [3]). In other words, we may say that bi-approximation semantics is a canonicity-friendly relational semantics for lattice-based logics. In addition, a Sahlqvist-type first-order definability for substructural logic was already shown in Suzuki [14]. Therefore, this completes the so-called Sahlqvist theorem for substructural and lattice-based logics.

Received April 23, 2013; accepted May 12, 2014

First published online April 20, 2016

2010 Mathematics Subject Classification: Primary 03G10, 03G25; Secondary 03G27

Keywords: lattice-based logics, relational semantics, canonicity

(C) 2016 by University of Notre Dame 10.1215/00294527-3542442 


\title{
The Distributivity on Bi-Approximation Semantics
}

\author{
Tomoyuki Suzuki
}

\begin{abstract}
In this paper, we give a possible characterization of the distributivity on bi-approximation semantics. To this end, we introduce new notions of special elements on polarities and show that the distributivity is first-order definable on bi-approximation semantics. In addition, we investigate the dual representation of those structures and compare them with bi-approximation semantics for intuitionistic logic. We also discuss that two different methods to validate the distributivity - by the splitters and by the adjointness — can be explicated with the help of the axiom of choice as well.
\end{abstract}

\section{Introduction}

Bi-approximation semantics is a universal relational-type semantics for substructural and lattice-based logics, not necessarily including distributive substructural logics such as orthonormal logic or lattice-based modal logics (see Suzuki [11]). Unlike other relational semantics for nondistributive lattice-based logics (see, e.g., Goldblatt [7], Hartonas [8], Hartonas and Dunn [9], Gehrke [5]; see also Restall [10]), the novelty of bi-approximation semantics is to reason not only about formulas but also sequents, that is, logical consequences, based on polarities. As bi-approximation semantics was introduced to explicate Ghilardi and Meloni's [6] canonicity methodology via relational-type structures, we can enjoy the canonicity results of latticebased logics in Suzuki [12] (cf. residuated frames in Galatos and Jipsen [3]). In other words, we may say that bi-approximation semantics is a canonicity-friendly relational semantics for lattice-based logics. In addition, a Sahlqvist-type first-order definability for substructural logic was already shown in Suzuki [14]. Therefore, this completes the so-called Sahlqvist theorem for substructural and lattice-based logics.

Received April 23, 2013; accepted May 12, 2014

First published online April 20, 2016

2010 Mathematics Subject Classification: Primary 03G10, 03G25; Secondary 03G27

Keywords: lattice-based logics, relational semantics, canonicity

(C) 2016 by University of Notre Dame 10.1215/00294527-3542442 


\title{
The Distributivity on Bi-Approximation Semantics
}

\author{
Tomoyuki Suzuki
}

\begin{abstract}
In this paper, we give a possible characterization of the distributivity on bi-approximation semantics. To this end, we introduce new notions of special elements on polarities and show that the distributivity is first-order definable on bi-approximation semantics. In addition, we investigate the dual representation of those structures and compare them with bi-approximation semantics for intuitionistic logic. We also discuss that two different methods to validate the distributivity - by the splitters and by the adjointness — can be explicated with the help of the axiom of choice as well.
\end{abstract}

\section{Introduction}

Bi-approximation semantics is a universal relational-type semantics for substructural and lattice-based logics, not necessarily including distributive substructural logics such as orthonormal logic or lattice-based modal logics (see Suzuki [11]). Unlike other relational semantics for nondistributive lattice-based logics (see, e.g., Goldblatt [7], Hartonas [8], Hartonas and Dunn [9], Gehrke [5]; see also Restall [10]), the novelty of bi-approximation semantics is to reason not only about formulas but also sequents, that is, logical consequences, based on polarities. As bi-approximation semantics was introduced to explicate Ghilardi and Meloni's [6] canonicity methodology via relational-type structures, we can enjoy the canonicity results of latticebased logics in Suzuki [12] (cf. residuated frames in Galatos and Jipsen [3]). In other words, we may say that bi-approximation semantics is a canonicity-friendly relational semantics for lattice-based logics. In addition, a Sahlqvist-type first-order definability for substructural logic was already shown in Suzuki [14]. Therefore, this completes the so-called Sahlqvist theorem for substructural and lattice-based logics.

Received April 23, 2013; accepted May 12, 2014

First published online April 20, 2016

2010 Mathematics Subject Classification: Primary 03G10, 03G25; Secondary 03G27

Keywords: lattice-based logics, relational semantics, canonicity

(C) 2016 by University of Notre Dame 10.1215/00294527-3542442 


\title{
The Distributivity on Bi-Approximation Semantics
}

\author{
Tomoyuki Suzuki
}

\begin{abstract}
In this paper, we give a possible characterization of the distributivity on bi-approximation semantics. To this end, we introduce new notions of special elements on polarities and show that the distributivity is first-order definable on bi-approximation semantics. In addition, we investigate the dual representation of those structures and compare them with bi-approximation semantics for intuitionistic logic. We also discuss that two different methods to validate the distributivity - by the splitters and by the adjointness — can be explicated with the help of the axiom of choice as well.
\end{abstract}

\section{Introduction}

Bi-approximation semantics is a universal relational-type semantics for substructural and lattice-based logics, not necessarily including distributive substructural logics such as orthonormal logic or lattice-based modal logics (see Suzuki [11]). Unlike other relational semantics for nondistributive lattice-based logics (see, e.g., Goldblatt [7], Hartonas [8], Hartonas and Dunn [9], Gehrke [5]; see also Restall [10]), the novelty of bi-approximation semantics is to reason not only about formulas but also sequents, that is, logical consequences, based on polarities. As bi-approximation semantics was introduced to explicate Ghilardi and Meloni's [6] canonicity methodology via relational-type structures, we can enjoy the canonicity results of latticebased logics in Suzuki [12] (cf. residuated frames in Galatos and Jipsen [3]). In other words, we may say that bi-approximation semantics is a canonicity-friendly relational semantics for lattice-based logics. In addition, a Sahlqvist-type first-order definability for substructural logic was already shown in Suzuki [14]. Therefore, this completes the so-called Sahlqvist theorem for substructural and lattice-based logics.

Received April 23, 2013; accepted May 12, 2014

First published online April 20, 2016

2010 Mathematics Subject Classification: Primary 03G10, 03G25; Secondary 03G27

Keywords: lattice-based logics, relational semantics, canonicity

(C) 2016 by University of Notre Dame 10.1215/00294527-3542442 


\title{
The Distributivity on Bi-Approximation Semantics
}

\author{
Tomoyuki Suzuki
}

\begin{abstract}
In this paper, we give a possible characterization of the distributivity on bi-approximation semantics. To this end, we introduce new notions of special elements on polarities and show that the distributivity is first-order definable on bi-approximation semantics. In addition, we investigate the dual representation of those structures and compare them with bi-approximation semantics for intuitionistic logic. We also discuss that two different methods to validate the distributivity - by the splitters and by the adjointness — can be explicated with the help of the axiom of choice as well.
\end{abstract}

\section{Introduction}

Bi-approximation semantics is a universal relational-type semantics for substructural and lattice-based logics, not necessarily including distributive substructural logics such as orthonormal logic or lattice-based modal logics (see Suzuki [11]). Unlike other relational semantics for nondistributive lattice-based logics (see, e.g., Goldblatt [7], Hartonas [8], Hartonas and Dunn [9], Gehrke [5]; see also Restall [10]), the novelty of bi-approximation semantics is to reason not only about formulas but also sequents, that is, logical consequences, based on polarities. As bi-approximation semantics was introduced to explicate Ghilardi and Meloni's [6] canonicity methodology via relational-type structures, we can enjoy the canonicity results of latticebased logics in Suzuki [12] (cf. residuated frames in Galatos and Jipsen [3]). In other words, we may say that bi-approximation semantics is a canonicity-friendly relational semantics for lattice-based logics. In addition, a Sahlqvist-type first-order definability for substructural logic was already shown in Suzuki [14]. Therefore, this completes the so-called Sahlqvist theorem for substructural and lattice-based logics.

Received April 23, 2013; accepted May 12, 2014

First published online April 20, 2016

2010 Mathematics Subject Classification: Primary 03G10, 03G25; Secondary 03G27

Keywords: lattice-based logics, relational semantics, canonicity

(C) 2016 by University of Notre Dame 10.1215/00294527-3542442 


\title{
The Distributivity on Bi-Approximation Semantics
}

\author{
Tomoyuki Suzuki
}

\begin{abstract}
In this paper, we give a possible characterization of the distributivity on bi-approximation semantics. To this end, we introduce new notions of special elements on polarities and show that the distributivity is first-order definable on bi-approximation semantics. In addition, we investigate the dual representation of those structures and compare them with bi-approximation semantics for intuitionistic logic. We also discuss that two different methods to validate the distributivity - by the splitters and by the adjointness — can be explicated with the help of the axiom of choice as well.
\end{abstract}

\section{Introduction}

Bi-approximation semantics is a universal relational-type semantics for substructural and lattice-based logics, not necessarily including distributive substructural logics such as orthonormal logic or lattice-based modal logics (see Suzuki [11]). Unlike other relational semantics for nondistributive lattice-based logics (see, e.g., Goldblatt [7], Hartonas [8], Hartonas and Dunn [9], Gehrke [5]; see also Restall [10]), the novelty of bi-approximation semantics is to reason not only about formulas but also sequents, that is, logical consequences, based on polarities. As bi-approximation semantics was introduced to explicate Ghilardi and Meloni's [6] canonicity methodology via relational-type structures, we can enjoy the canonicity results of latticebased logics in Suzuki [12] (cf. residuated frames in Galatos and Jipsen [3]). In other words, we may say that bi-approximation semantics is a canonicity-friendly relational semantics for lattice-based logics. In addition, a Sahlqvist-type first-order definability for substructural logic was already shown in Suzuki [14]. Therefore, this completes the so-called Sahlqvist theorem for substructural and lattice-based logics.

Received April 23, 2013; accepted May 12, 2014

First published online April 20, 2016

2010 Mathematics Subject Classification: Primary 03G10, 03G25; Secondary 03G27

Keywords: lattice-based logics, relational semantics, canonicity

(C) 2016 by University of Notre Dame 10.1215/00294527-3542442 


\title{
The Distributivity on Bi-Approximation Semantics
}

\author{
Tomoyuki Suzuki
}

\begin{abstract}
In this paper, we give a possible characterization of the distributivity on bi-approximation semantics. To this end, we introduce new notions of special elements on polarities and show that the distributivity is first-order definable on bi-approximation semantics. In addition, we investigate the dual representation of those structures and compare them with bi-approximation semantics for intuitionistic logic. We also discuss that two different methods to validate the distributivity - by the splitters and by the adjointness — can be explicated with the help of the axiom of choice as well.
\end{abstract}

\section{Introduction}

Bi-approximation semantics is a universal relational-type semantics for substructural and lattice-based logics, not necessarily including distributive substructural logics such as orthonormal logic or lattice-based modal logics (see Suzuki [11]). Unlike other relational semantics for nondistributive lattice-based logics (see, e.g., Goldblatt [7], Hartonas [8], Hartonas and Dunn [9], Gehrke [5]; see also Restall [10]), the novelty of bi-approximation semantics is to reason not only about formulas but also sequents, that is, logical consequences, based on polarities. As bi-approximation semantics was introduced to explicate Ghilardi and Meloni's [6] canonicity methodology via relational-type structures, we can enjoy the canonicity results of latticebased logics in Suzuki [12] (cf. residuated frames in Galatos and Jipsen [3]). In other words, we may say that bi-approximation semantics is a canonicity-friendly relational semantics for lattice-based logics. In addition, a Sahlqvist-type first-order definability for substructural logic was already shown in Suzuki [14]. Therefore, this completes the so-called Sahlqvist theorem for substructural and lattice-based logics.

Received April 23, 2013; accepted May 12, 2014

First published online April 20, 2016

2010 Mathematics Subject Classification: Primary 03G10, 03G25; Secondary 03G27

Keywords: lattice-based logics, relational semantics, canonicity

(C) 2016 by University of Notre Dame 10.1215/00294527-3542442 


\title{
The Distributivity on Bi-Approximation Semantics
}

\author{
Tomoyuki Suzuki
}

\begin{abstract}
In this paper, we give a possible characterization of the distributivity on bi-approximation semantics. To this end, we introduce new notions of special elements on polarities and show that the distributivity is first-order definable on bi-approximation semantics. In addition, we investigate the dual representation of those structures and compare them with bi-approximation semantics for intuitionistic logic. We also discuss that two different methods to validate the distributivity - by the splitters and by the adjointness — can be explicated with the help of the axiom of choice as well.
\end{abstract}

\section{Introduction}

Bi-approximation semantics is a universal relational-type semantics for substructural and lattice-based logics, not necessarily including distributive substructural logics such as orthonormal logic or lattice-based modal logics (see Suzuki [11]). Unlike other relational semantics for nondistributive lattice-based logics (see, e.g., Goldblatt [7], Hartonas [8], Hartonas and Dunn [9], Gehrke [5]; see also Restall [10]), the novelty of bi-approximation semantics is to reason not only about formulas but also sequents, that is, logical consequences, based on polarities. As bi-approximation semantics was introduced to explicate Ghilardi and Meloni's [6] canonicity methodology via relational-type structures, we can enjoy the canonicity results of latticebased logics in Suzuki [12] (cf. residuated frames in Galatos and Jipsen [3]). In other words, we may say that bi-approximation semantics is a canonicity-friendly relational semantics for lattice-based logics. In addition, a Sahlqvist-type first-order definability for substructural logic was already shown in Suzuki [14]. Therefore, this completes the so-called Sahlqvist theorem for substructural and lattice-based logics.

Received April 23, 2013; accepted May 12, 2014

First published online April 20, 2016

2010 Mathematics Subject Classification: Primary 03G10, 03G25; Secondary 03G27

Keywords: lattice-based logics, relational semantics, canonicity

(C) 2016 by University of Notre Dame 10.1215/00294527-3542442 